\title{
eJRIEPS
}

Ejournal de la recherche sur l'intervention en éducation physique et sport

Hors-série $N^{\circ} 1 \mid 2015$

Quelques aspects théoriques de la didactique des sports collectifs. Préparation aux concours de recrutement

\section{Des éléments de conclusion}

Jean-Francis Gréhaigne

\section{(2) OpenEdition}

Journals

Édition électronique

URL : http://journals.openedition.org/ejrieps/1326

DOI : 10.4000/ejrieps. 1326

ISSN : 2105-0821

Éditeur

ELLIADD

Référence électronique

Jean-Francis Gréhaigne, «Des éléments de conclusion », eJRIEPS [En ligne], Hors-série N 1 | 2015, mis en ligne le 01 décembre 2015, consulté le 10 octobre 2019. URL : http://journals.openedition.org/ ejrieps/1326 ; DOI : 10.4000/ejrieps.1326

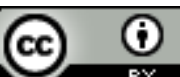

La revue eJRIEPS est mise à disposition selon les termes de la Creative Commons Attribution 4.0 International License. 
eJRIEPS Hors série n¹ Décembre 2015

\section{Des éléments de conclusion}

\section{Jean-Francis Gréhaigne}

\section{Professeur des Universités retraité, Université Bourgogne-Franche-Comté}

Dans le développement de la lutte contre l'analphabétisme, le choix de l'apprentissage au travers de la compréhension est sans aucun doute un des facteurs les plus importants de n'importe quel système éducatif dans le monde entier. L'enseignement implique le développement du langage et des compétences sur les façons appropriées de penser en vue de la résolution de problèmes dans toutes les disciplines. Le dialogue en classe a beaucoup à voir avec ce développement, car le discours joue un rôle central et de façon critique importante à tous les niveaux d'enseignement. Ainsi, la littératie est à la mode : nous définirons celle-ci comme "l'aptitude à comprendre et à utiliser les informations dans la vie courante, à l'école, au travail et dans sa vie de loisir en vue d'atteindre des buts personnels et d'étendre ses connaissances et ses capacités ». L'enjeu de la formation est pour les élèves d'apprendre à réfléchir, à élaborer consciemment des réponses aux problèmes qu'on leur pose quelle que soit l'activité proposée. Cet objectif d'ordre méthodologique doit être poursuivi, pour ce qui nous concerne, en étroite relation avec des objectifs liés à l'acquisition de savoirs spécifiques en éducation physique. Le but de l'apprentissage est, alors, la constitution de connaissances et de compétences motrices dans lesquelles la composante mentale - représentations, planifications et stratégies de l'action motrice - est essentielle. C'est pourquoi nous considérons qu'un élève a appris quand il ou elle a réussi et est capable d'énoncer les raisons de sa réussite en formulant par exemple des règles d'action.

Le modèle de fonctionnement et de développement du joueur de sport collectif qui vient d'être esquissé met l'accent sur la pluralité des processus et sur la nature des relations qu'ils entretiennent dans le fonctionnement du sujet. Cette vision du joueur semble universelle dans la mesure où le répertoire des processus est le même pour tous les sujets. Elle est différentielle dans la mesure où la hiérarchie entre les éléments du système de variables est changeante selon les affrontements, les équipes et / ou selon les individus. Ce type de fonctionnement fait de l'interaction entre ces processus et des modes de traitement une source du développement du joueur. Cette approche pluraliste admet, par construction, qu'un même problème puisse être résolu par des processus différents. Elle ne situe pas les différences entre joueurs seulement dans les propriétés 


\section{eJRIEPS Hors série n¹ Décembre 2015}

des situations mais aussi dans les modes de représentation et de traitement qu'elle sollicite.

En définitive, les notions de disponibilité, d'interactions, et de mises en relation constituent, nous semble-t-il, des éléments décisifs pour appréhender le jeu et le développement du joueur.

Les sports collectifs à l'école restent un formidable outil pour travailler en groupe et apprendre à gérer des trajets et des trajectoires plus ou moins aléatoires de mobiles dans des conditions d'urgence décisionnelles. Les connaissances reposent sur des compétences motrices et des règles d'action ainsi que des règles de gestion de l'organisation du jeu. Les configurations du jeu demeurent un outil irremplaçable pour comprendre et analyser, au mieux, l'évolution des rapports d'opposition. Le débat d'idées donne la possibilité aux élèves de partager leurs expériences collectives dans le cadre d'un rapport de forces en regard des configurations momentanées du jeu apparues. Mais, offrir aux élèves la meilleure éducation physique possible implique bien évidemment de nombreux autres facteurs. La gestion du temps par exemple car le temps pour décider et le temps pour faire sont variables sous pression temporelle. La tactique suppose chez les joueurs des capacités à utiliser pour l'action, les déterminants et les aléas extérieurs en un mot jouer avec le temps. L'opposition et ses concepts associés constituent bien l'essence des sports collectifs. Les rapports d'opposition produisant des configurations du jeu renferment de nombreuses réponses possibles plus ou moins adaptées au rapport de forces en cours. La primauté accordée au jeu recouvre deux aspects complémentaires : tout d'abord l'activité ludique qui constitue une dimension importante de l'éducation physique, ensuite une activité de compétition où le résultat est important mais le fait de se confronter aux autres engendre le progrès.

Le joueur pour répondre aux problèmes posés par le jeu, doit posséder, une intelligence de jeu bien particulière. Dans une rencontre de sport collectif, les actions individuelles de tous joueurs construisent une capacité collective propre, c'est-à-dire irréductible à celle de ses membres. Cette trame dynamique de transformation est un ensemble humain structuré qui coordonne ou perturbe les actions menées par l'ensemble des participants à l'aide de mécanismes d'interactions relativement stables. Ceci entraîne une circularité des problèmes posés à l'équipe, au plan de l'organisation du jeu comme au plan de la distribution des joueurs. La notion de rapport de forces, entre les deux équipes en présence, permet également de mettre en évidence la notion de pression sur tel ou tel maillon du jeu pour rompre l'équilibre des forces en présence dans les affrontements 


\section{eJRIEPS Hors série n¹ Décembre 2015}

momentanés. Ainsi, on vise à créer un déséquilibre favorable en prenant des risques pour prendre un avantage pour aller marquer un point. Ou bien on privilégie la sécurité en préservant temporairement une certaine stabilité défensive mais en laissant l'initiative du jeu aux adversaires.

Enfin, une autre difficulté importante consiste à savoir comment les enseignants enseignent et surtout comment les élèves apprennent. Les chercheurs ont besoin d'approfondir les voies d'apprentissage et de décrire, chez les élèves, les processus cognitifs et la construction de l'expérience collective qui se produisent pendant le jeu. En faisant cela, on pourra mieux comprendre les mécanismes qui influencent les relations entre l'acquisition des connaissances et les performances motrices. Un environnement d'apprentissage efficace crée nécessairement des conditions d'enseignement efficientes.

Enfin, au sein de notre groupe de recherche, pour produire ces données, nous avons organisé le travail autour de trois principes. Tout d'abord, nous mettons à l'épreuve des faits, en développant une approche quasi-expérimentale. II s'agit de traiter de vrais problèmes qui se posent à de véritables acteurs, en tentant d'apporter des réponses. Dans un deuxième point, il faut souligner que nous sommes confrontés à des savoirs complexes et nous ne pouvons pas uniquement copier les modèles issus des autres sciences ou de la pratique de haut niveau. Cette démarche peut impliquer l'élaboration de concepts nouveaux pour articuler les connaissances issues de différents champs mais aussi que les concepts importés soient travaillés, adaptés et acquièrent ainsi une signification nouvelle au regard des besoins. Cette posture épistémologique est à l'opposé de la conception et de l'exportation des "good practices " chères aux anglophones qui laissent la culture locale et les élèves sur le bord du chemin au profit d'un soit disant « prêt à porter didactique universel ».

Somme toute, nous avons le souci de produire des outils qui soient utilisables et adaptables par tous et qui mettent en avant l'émergence, la construction et la stabilisation des connaissances et compétences motrices dans la communauté scolaire. Donc, nous attachons du prix à la diffusion des résultats de notre travail. Alors, les propositions que nous avons faites dans ce numéro "hors-série ", s'inscrivent dans cette logique de production à propos de la didactique et de la modélisation du jeu en sport collectif. 